\title{
Safety and Efficacy of NOACs and Warfarin in Singapore: Are They Really Equivalent?
}

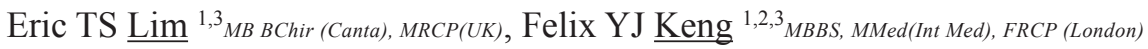

In this issue of the Annals, an important subject has been put into perspective, that of oral anticoagulation (OAC) for non-valvular AF. ${ }^{1}$ Although there has been a wealth of literature regarding this topic, little is known in the Singapore population with respect to the benefits and drawbacks associated with the different available OACs. In this article, Wong et al. ${ }^{1}$ are to be congratulated for a very well-conducted, albeit retrospective comparison of 3 major non-vitamin $\mathrm{K}$ oral anticoagulants (apixaban, rivaroxaban and dabigatran) as compared to warfarin.

Key features of this important study included: (1) detailed characterisation of subjects enabling calculation of $\mathrm{CHA}_{2} \mathrm{DS}_{2}-\mathrm{VASc}$ and HAS-BLED scores acting as covariates for subsequent analyses, (2) calculation of time-in-therapeutic range (TTR) to assess quality of warfarin anticoagulation, and (3) detailed scrutiny of pharmacy dispensing records to assess compliance to novel oral anticoagulation (NOAC) prescription as quantified by the medication possession rate (MPR). Furthermore, incomplete data was present in only $1 \%$ of the subjects and follow-up data was available in $100 \%$. This degree of rigour is uncommon in similar retrospective studies; however, it is offset by the relatively low numbers of enrolled subjects and clinical events associated with a single-centre study.

Several notable findings arise from this study that merit detailed discussion. Firstly, the TTR of $68.8 \%$ reported in this real-world study is remarkably high, and is similar or exceeds that reported under trial settings. For example, the country-specific TTR in the Randomised Evaluation of Long-Term Anticoagulation Therapy (RE-LY) trial comparing dabigatran and warfarin was $68 \%$ for Singapore and this TTR exceeds all other Asian countries in the RE-LY trial. ${ }^{2}$ We should therefore expect good outcomes in those patients receiving warfarin anticoagulation.

By contrast, NOAC compliance seems relatively low, as assessed by the MPR. There is still a relative paucity of high-quality literature surrounding NOAC compliance., What constitutes a good MPR? There is currently no data that relates the minimum compliance needed for a NOAC to be clinically efficacious. Some early papers as well as a meta-analysis report high real-life NOAC compliance rates, with MPR80 (i.e. proportion of patients with MPR values exceeding $80 \%$ ) in the $70-75 \%$ range. ${ }^{4}$ However, in this study, the MPR80 was only $45-59 \%$. We should point out that this MPR range is similar to that reported for many drugs used in other chronic diseases. ${ }^{5}$

In terms of the outcome measures, these should therefore be interpreted in light of the simultaneously high TTR but low MPR values. Excluding dabigatran (due to the low enrolled numbers), the chief finding was that rivaroxaban and apixaban performed similarly to warfarin as assessed by stroke, major bleeding and overall bleeding metrics. The takeaway message from this study is that, in fact, NOAC performs very well even outside of clinical trials and even with suboptimal compliance. We can expect the NOAC advantage over warfarin to be amplified in hospitals and countries where excellent TTR cannot be achieved.

What about the finding that NOACs exhibited significantly shorter time to thromboembolic events as compared to the warfarin group? Given the relatively low number of events ( 25 in total across all subjects, and which included the softer end-point of transient ischaemic attack), we think this should be interpreted with caution and regarded as a hypothesis-generating finding only. The authors suggest that a possible reason for this finding is the low apparent compliance for NOACs, coupled with their relatively low elimination half-lives (7-11 hours, 10-14 hours and 14-17 hours for rivaroxaban, apixaban and dabigatran, respectively). To explore this further, a comparison of the MPR in patients experiencing events versus those without would be interesting but not included in the study-this comparison may not have been possible or meaningful in a study of this size.

\footnotetext{
${ }^{1}$ National Heart Centre, Singapore

${ }^{2}$ Yong Loo Lin School of Medicine, National University of Singapore, Singapore

${ }^{3}$ Duke-NUS Medical School, Singapore

Address for correspondence: Dr Eric TS Lim, National Heart Centre Singapore, 5 Hospital Drive, Singapore 169609.

Email: eric.lim.t.s@singhealth.com.sg
} 
While compliance is an obvious explanation for this aspect of subpar performance of NOACs, there are other alternative explanations: $17 \%$ of rivaroxaban patients were underdosed; and underdosing of NOACs has been reported to be common (e.g. underdosing of both rivaroxaban and apixaban exceeded $50 \%$ in the Korean National Health Insurance Service database). This should in theory lead to suboptimal NOAC performance although this has not always been reported to be so.

Another intriguing explanation offered by the authors relates to the finding that serum rivaroxaban levels after taking a single dose of rivaroxaban were found to be lower in Asians versus non-Asians living in Singapore. ${ }^{6}$ If so, then the expectation would be that the thromboembolic protection offered by rivaroxaban would also be reduced and suggests important ethnicity-specific differences in drug metabolism. This pharmacokinetic study is relatively small and further validation is required, particularly as the Rivaroxaban Once Daily Oral Direct Factor Xa Inhibition Compared with Vitamin K Antagonism for Prevention of Stroke and Embolism Trial in Atrial Fibrillation (ROCKET AF) trial that compared rivaroxaban to warfarin observed similar relative efficacy and safety between East Asians and non-East Asians. ${ }^{7}$ This finding also goes against the J-ROCKET AF study, ${ }^{8}$ which was a prospective, randomised, double-blind, phase III trial of 1,280 patients similar in design to the ROCKET AF trial but with all participants drawn from Japan.This trial found that the $15 \mathrm{mg}$ dose of rivaroxaban was non-inferior to warfarin in terms of protection from stroke and systemic embolism.

To conclude, Wong et al. have conducted an important study that sheds insight into anticoagulation using both NOAC and warfarin in Singapore. It suggests avenues for further exploration, at a scientific level (e.g. possible ethnic variations in the pharmacokinetics of NOAC metabolism), as well as at a service level (e.g. the targeting of compliance to NOACs as a means of improving NOAC anticoagulation outcomes). We look forward to further publications in these areas.

\section{REFERENCESS}

1. Tiew, WJ, Wong LX, Tan VH, et al. A Real-world Experience of the Safety and Efficacy of Non-vitamin K Oral Anticoagulants Versus Warfarin in Patients with Non-valvular Atrial Fibrillation - A Single-centre Retrospective Cohort Study in Singapore. Ann Acad Med Singap 2020;49:838-47.

2. Lars W, Yusuf S, Ekekowitz MD, et al. Efficacy and safety of dabigatran compared with warfarin at different levels of international normalised ratio control for stroke prevention in atrial fibrillation: an analysis of the RE-LY trial. Lancet 2010;376:975-83,

3. Rodriguez-Bernal CL, Garcia-Sempere A, Hurtado I, et al. Realworld adherence to oral anticoagulants in atrial fibrillation patients: a study protocol for a systematic review and meta-analysis. BMJ Open 2018;8:e025102.

4. Salmasi S, Loewen PS, Tandun R, et al. Adherence to oral anticoagulants among patients with atrial fibrillation: a systematic review and meta-analysis of observational studies. BMJ Open 2020;10:e034778.

5. Yeaw J, Benner JS, Walt JG, et al. Comparing adherence and persistence across 6 chronic medication classes. J Manag Care Pharm $2009 ; 15: 728-40$

6. Ho NT, Goh JJN, Aw JWX, et al. Comparison of rivaroxaban concentrations between Asians and Caucasians and their correlation with PT/INR. J Thromb Thrombolysis 2018;46:541-8.

7. Ng Tsai HO, Goh JJN, Aw JWX, et al. Comparison of rivaroxaban concentrations between Asians and Caucasians and their correlation with PT/INR. J Thromb Thrombolysis 2018;46:541-8.

8. Hori M, Matsumoto M, Tanahashi N, et al. Rivaroxaban vs. Warfarin in Japanese Patients With Atrial Fibrillation, The J-ROCKET AF Study. Circ J 2012;76:2104-11. 